\title{
Fault detection reduced-order filter design for discrete-time Markov jump system with deficient transition information
}

\author{
Lihong Rong ${ }^{1,2}$, Xiuyan Peng ${ }^{1 *}$ and Biao Zhang ${ }^{1}$
}

\begin{abstract}
The paper addresses the fault detection (FD) problem for a class of discrete-time Markov jump linear systems (MJLSs) with deficient transition rates, which simultaneously considers the totally known, partly unknown, and uncertain transition rates. Then, in accordance with the linear matrix inequality (LMI) method and the convexification techniques, a sufficient condition for the existence of FD reduced-order filter over MJLSs with deficient transition information is obtained, which can ensure the error augmented system with the FD reduced-order filter is stochastically stable. In addition, a performance index is given to enhance the robustness of the residual system against deficient transition information and external disturbance, such that the error between the fault and the residual is made as small as possible to reinforce the faults sensitivity. Finally, an illustrative example is employed to show the effectiveness of the proposed design approach.
\end{abstract}

Keywords: Markov jump linear systems, Fault detection, Deficient transition rate, Reduced-order filter

\section{Introduction}

During the past decades, Markov jump linear systems (MJLSs) have been received extensive interests in many engineering fields, such as energy system, solar thermal power generation system, networked control system, manufacturing system, financial market system $[1,2]$. Many important results have been reported, such as a number of studies on the Markovian jump system on the filter design [3-5], controller design [6], output feedback control [7-10], stability analysis and synthesis [11-13]. In fact, MJLSs are very appropriate to dynamical model systems whose property is subject to random sudden variant due to abrupt external disturbance, shifting of the action spots of a nonlinear system, and repairs of components, thus, in order to ensure the nonlinear system stochastically exponentially stable, the author in [9] proposed a Markovian Lyapunov functional which was been successfully used in the nonlinear systems. In essence, the transition rates (TRs) in the MJLSs are considerable up to now. A large number of traditional analysis and design

*Correspondence: pxygll@sina.com

${ }^{1}$ College of Automation, Harbin Engineering University, Harbin 150001, China Full list of author information is available at the end of the article results have been reported on condition that the exactly known the TRs in the MJLSs $[14,15]$. However, it should be pointed that all the mode transition rates can not be acquired totally in lots of engineering plants. That is to say, for a majority of MJLSs, there are three types of transition cases for the MJLSs, e.g., some are known, some are unknown, and others are polytopic uncertain TRs $[16,17]$. On the other hand, in many published papers, the unknown TRs and polytopic uncertain TRs in MJLSs have been taken into account separatedly. In reality, in a lot of actual conditions, there are the uncertain TRs and unknown TRs in MJLSs synchronously. To mention a few, the author in [2] investigated continuous-time Markovian jump problems with deficient transition descriptions. In [18], an $H_{\infty}$ filtering was developed for the two dimensional continuous time MJLSs with deficient transition descriptions. In this way, it is more rational and general to research on the analysis and synthesis of MJLSs with the totally known, uncertain and partly unknown TRs concurrently, which is the main motivation to do my research.

On another research frontier, the fault detection and isolation techniques have gotten a great number of attention in the academic research and practical application 
because of the increasing demand for improving the system reliability and the safety of modern plant operations and reducing costs (see [19]-[29]). The basic design idea of FD is to use the effective methods to generate a residual signal and to construct a common diagnostic residual evaluation function to compare with a beforehand threshold, then an alarm of fault is generated when the value of system residual is larger than the threshold [30, 31]. Hence, in the process of fault detection, residual generation is a very important step, based on this, there are many basic approaches are provided to generate robust residuals that are sensitive to faults, while insensitive to unknown input and noise. In the existing methods, which have been proposed to detect faults, such as fullstate observer-based methods [32], optimization-based approach [33], parity relations approach [34], unknown input observers [35, 36], system identification methods $[37,38]$, nonlinear approach $[39,40]$, artificial intelligence techniques [41, 42], discrete event systems and hybrid systems [43-46], the fault detection filter method is the most favoured method. However, in many practical applications, high-order models are frequently used to describe physical systems. This brings many difficulties in design of the corresponding FD filter in order to detect faults in a timely way. Moreover, to the knowledge of the authors, there are few results have been reported in the literature on the high-efficiency FD reduced-order filter design. This motivates us to study this work in order to reduce the complexity, computation time of the FD filter design process and save storage space, so as to improve the efficiency of the fault detection, which has great potential in practical applications.

In this paper, the chief aim is to design the FD reducedorder filter for a family of discrete-time MJLSs with deficient TRs, which is more general. By satisfying some performance indexes, the susceptibility to malfunction and the robustness against interference are both enhanced on residual outputs. Through the constructing of the residual generator, the FD design scheme is converted to an $H_{\infty}$ filtering problem in order that the error between residual and malfunction is minimized at the $H_{\infty}$ level. Then, the sufficient condition for the existence of the FD filter for the represented systems is obtained via linear matrix inequalities. Finally, a numerical example is presented to show the effectiveness and potential of the developed theoretical result. In fact, the problem of fault detection for MJLSs with deficient TRs should meet many requirements of detection performance and Markov jump process, which leads to the increase difficulty of filter design. Therefore, to the best knowledge of the author, the research on the fault detection reduced-order filters for Markov jump system with deficient transition information is relatively few, which is the third motivation for this research.
The remainder of this paper is organized as follows. In Section 2, the mathematical model of the system is formulated, and then, many preliminary results are shown. The sufficient condition of FD filter for the underlying system is established in Section 3. In Section 4, a simulation example is presented to point out the effectiveness of the proposed approach.

Notations. $\mathbb{R}^{n}$ denotes the $\mathrm{n}$-dimensional Euclidean space, $\mathbb{R}^{m \times n}$ denotes the set of all $m \times n$ real matrices, and $N$ represents a positive integer. The notation $P>0$, where $P$ is the symmetric matrix, which means that $P$ is positive definite. In a symmetric matrix, $*$ represents the symmetric element. $\|\cdot\|$ denotes the Euclidean vector norm of a vector, $l_{2}[0, \infty)$ is the space of all square summable vector functions for $\omega=\{\omega(k)\} \in l_{2}[0, \infty)$, and its norm is given by $\|\omega\|_{2}=\sqrt{\sum_{k=0}^{\infty}\|\omega\|^{2}}$. The mathematical expectation operator is denoted as $E[\cdot]$, and $\|Z\|_{\varepsilon_{2}}=\sqrt{E\left[\sum_{k=0}^{\infty}\|Z\|^{2}\right]}$ indicates its norm.

\section{Problem formulation}

Consider the following discrete-time MJLS on a complete rate space of the form:

$$
\begin{aligned}
& x_{k+1}=A\left(r_{k}\right) x_{k}+B\left(r_{k}\right) u_{k}+E\left(r_{k}\right) \omega_{k}+F\left(r_{k}\right) f_{k}, \\
& y_{k}=C\left(r_{k}\right) x_{k}+D\left(r_{k}\right) \omega_{k}+H\left(r_{k}\right) f_{k},
\end{aligned}
$$

where $x_{k} \in \mathbb{R}^{n_{x}}$ represents the plant state, $u_{k} \in \mathbb{R}^{n_{u}}$ is the known control input, $\omega_{k} \in \mathbb{R}^{n_{d}}$ is the exogenous disturbance signal, $f_{k} \in \mathbb{R}^{n_{f}}$ is the fault signal to be detected, $y_{k} \in \mathbb{R}^{n_{y}}$ is the controlled output, $u_{k}, \omega_{k}$ and $f_{k}$ are assumed to belong to $l_{2}[0, \infty) .\left\{r_{k}, k>0\right\}$ is a discretetime homogeneous Markov chain, which takes values in a finite set $S=\{1,2, \ldots, n\}$ with mode transition rates (TRs) $\operatorname{Prob}\left(r_{k+1}=j \mid r_{k}=i\right)=\lambda_{i j}$, where $\lambda_{i j} \geq 0$, for all $i, j \in S$ and $\Sigma_{j=1}^{N} \lambda_{i j}=1$. For $r_{k}=i, i \in S$, the system matrices of the $i$-th mode are denoted by $\left(A_{i}, B_{i}, C_{i}, D_{i}\right)$, which are known real matrices. In this paper, system (1) is assumed randomly stable, which is a precondition for model design.

Moreover, the TRs of the Markov process be regarded as being polytopic uncertain and partly available; in other words, the transition rate matrix (TRM) $\Lambda=\left\{\lambda_{i j}\right\}$ is deemed to belong to a known polytope $P_{\Lambda}$ with vertices $\Lambda_{s}$.

$$
P_{\Lambda}:=\left\{\Lambda \mid \Lambda=\sum_{s=1}^{M} \alpha_{s} \Lambda_{s} ; \alpha_{s} \geq 0, \sum_{s=1}^{M} \alpha_{s}=1\right\},
$$

where vertices $\Lambda_{s}=\left[\lambda_{i j}\right]_{N \times N}, i, j \in S, I=1,2 \cdots M$ are still given TRM containing unknown and uncertain 
factors. For example, for system (1) with four variation modes, the TRM may be as:

$$
\left[\begin{array}{llll}
\tilde{\lambda}_{11} & \lambda_{12} & \tilde{\lambda}_{13} & \hat{\lambda}_{14} \\
\hat{\lambda}_{21} & \hat{\lambda}_{22} & \lambda_{23} & \hat{\lambda}_{24} \\
\hat{\lambda}_{31} & \lambda_{32} & \hat{\lambda}_{33} & \lambda_{34} \\
\lambda_{41} & \tilde{\lambda}_{42} & \hat{\lambda}_{43} & \lambda_{44}
\end{array}\right]
$$

where the superscripts labeled with " " and " " represent the polytopic uncertainties and unknown TRs, separately, and the others are known TRs. In order to make the notational more clearly, for all $i \in S$, we denote $S=S_{k}^{(i)} \cup S_{u c}^{(i)} \cup$ $S_{u k}^{(i)}$ as follows:

$$
\begin{aligned}
& S_{k}^{(i)}:=\left\{j: \lambda_{i j} \text { is known }\right\}, \\
& S_{u c}^{(i)}:=\left\{j: \tilde{\lambda}_{i j} \text { is uncertain }\right\}, \\
& S_{u k}^{(i)}:=\left\{j: \hat{\lambda}_{i j} \text { is unknown }\right\} .
\end{aligned}
$$

Also, we define $\lambda_{u k}^{(i s)}:=\sum_{j \in S_{u k}^{(i)}} \hat{\lambda}_{i j}=1-\sum_{j \in S_{k}^{(i)}} \lambda_{i j}-\sum_{j \in S_{u c}^{(i)}} \tilde{\lambda}_{i j}^{(i s)}$.

Remark 1. The transition rates of the MJLSs $\left\{r_{k}, k \geq 0\right\}$ have been universally assumed to be some known, some unknown, and some uncertain within given intervals. Hence, the TRM considered in this article is more natural to the MJLSs, which includes the previous three cases. Then, we are interested in designing an FD filter for the underlying system, and its desired structure is considered to be:

$$
\begin{aligned}
& \hat{x}_{k+1}=A_{F}\left(r_{k}\right) \hat{x}_{k}+B_{F}\left(r_{k}\right) y_{k}, \\
& \hat{r}_{k}=C_{F}\left(r_{k}\right) \hat{x}_{k}+D_{F}\left(r_{k}\right) y_{k},
\end{aligned}
$$

where, $\hat{x}_{k} \in \mathbb{R}^{n}$ is the state estimation of filter, $\hat{r}_{k} \in \mathbb{R}^{f}$ is the residual, and $A_{F}\left(r_{k}\right), B_{F}\left(r_{k}\right), C_{F}\left(r_{k}\right)$, $D_{F}\left(r_{k}\right)$, and $\forall r_{k} \in \mathrm{I}$ are the matrices to be calculated.

Define $\tilde{x}_{k}:=\left[\begin{array}{cc}x_{k}^{T} & \hat{x}_{k}^{T}\end{array}\right]^{T}, \quad e_{k}:=\hat{r}_{k}-f_{k}$. Then, by augmenting (1) and (4), the error augmented system is obtained as follows:

$$
\begin{aligned}
& \tilde{x}_{k+1}=\tilde{A}\left(r_{k}\right) \tilde{x}_{k}+\tilde{B}\left(r_{k}\right) \psi_{k}, \\
& e_{k}=\tilde{C}\left(r_{k}\right) \tilde{x}_{k}+\tilde{D}\left(r_{k}\right) \psi_{k},
\end{aligned}
$$

where, $\psi_{k}=\left[\begin{array}{lll}u_{k}^{T} & \omega_{k}^{T} & f_{k}^{T}\end{array}\right]^{T}$ and

$$
\begin{aligned}
& \tilde{A}\left(r_{k}\right)=\left[\begin{array}{cc}
A\left(r_{k}\right) & 0 \\
B_{F}\left(r_{k}\right) C\left(r_{k}\right) & A_{F}\left(r_{k}\right)
\end{array}\right], \\
& \tilde{B}\left(r_{k}\right)=\left[\begin{array}{ccc}
B\left(r_{k}\right) & E\left(r_{k}\right) & F\left(r_{k}\right) \\
0 & B_{F}\left(r_{k}\right) D\left(r_{k}\right) & B_{F}\left(r_{k}\right) H\left(r_{k}\right)
\end{array}\right], \\
& \tilde{C}\left(r_{k}\right)=\left[D_{F}\left(r_{k}\right) C\left(r_{k}\right) C_{F}\left(r_{k}\right)\right], \\
& \tilde{D}\left(r_{k}\right)=\left[\begin{array}{ll}
0 & D_{F}\left(r_{k}\right) D\left(r_{k}\right) D_{F}\left(r_{k}\right) H\left(r_{k}\right)-I
\end{array}\right] .
\end{aligned}
$$

In fact, the error augmented system (5) is also an MJLS with deficient TRM in (3). Now, to describe the main objective of this article more precisely, let us recommend the following definitions for system (5), which are necessary for the later progress.

Definition 1. [16] A discrete-time stochastic system (5) is said to be stochastically stable if for $\psi_{k}=0, k \geq 0$ and every initial condition $\tilde{x}_{0} \in \mathbb{R}^{n_{x}}$ and $r_{0} \in S$ the following holds: $E\left\{\sum_{k=0}^{\infty}\left\|\tilde{x}_{k}\right\|^{2} \mid \tilde{x}_{0}, r_{0}\right\}<\infty$

Definition 2. [6] Given the disturbance input $\omega_{k} \in$ $l_{2}[0, \infty)$, a scalar $\gamma>0$, system (5) is stochastically stable and has an $H_{\infty}$ performance index $\gamma$ if the following two conditions are satisfied:

1) When $\omega_{k}=0, k \geq 0$, system (5) is stochastically stable in the sense of Definition 1.

2) When $\omega_{k} \neq 0, k \geq 0$, under zero initial conditions, the following inequality holds:

$$
E\left\{\sum_{K=0}^{\infty}\left\|e_{k}\right\|^{2}\right\}<\gamma^{2} E\left\{\sum_{K=0}^{\infty}\left\|\omega_{k}\right\|^{2}\right\}
$$

As a consequence, the main purposes of this paper are to determine matrices $\left\{A_{F}\left(r_{k}\right), B_{F}\left(r_{k}\right), C_{F}\left(r_{k}\right), D_{F}\left(r_{k}\right)\right\}$ in system (4), such that the augmented error system (5) is randomly stable with a reliable $H_{\infty}$ performance level $\gamma$ with deficient transition information. Finally, the discretetime MJLS (1) will be assumed to be stable in the end. Moreover, in order to detect the fault $f_{k}$, the residual evaluation function is designed as $J\left(\hat{r}_{g}\right)=\sqrt{\sum_{g=k_{0}}^{k_{0}+L} \hat{r}_{g}^{T} \hat{r}_{g}}$, where $k_{0}$ denotes the initial evaluation time instant. The fault $f_{k}$ can be detected by the following steps.

i) Select a threshold $J_{\text {th }} \triangleq \sup _{d \in l_{2}, f=0} E\left[J\left(\hat{r}_{k}\right)\right]$.

ii) Based on the above result, the fault $f_{k}$ can be detected by comparing $J\left(\hat{r}_{k}\right)$ and $J_{\text {th }}$.

iii) When $J\left(\hat{r}_{k}\right) \geq J_{\text {th }}$, there are some faults, we should give an alarm; when $J\left(\hat{r}_{k}\right)<J_{\text {th }}$, there are no faults.

Before proceeding further, we give the following lemma on the $H_{\infty}$ performance analysis of system (5) with completely known TRs, which will be used in the derivation of our main results.

Lemma 1. (see([3])) Given the disturbance input $\omega_{k} \in$ $l_{2}[0, \infty)$, for the MJLS (5) with completely known TRs and a given scalar $\gamma>0$, if the coupled inequalities

$$
\left[\begin{array}{cc}
\tilde{A}_{i}^{T} \eta_{i} \tilde{A}_{i}-P_{i}+\tilde{C}_{i}^{T} \tilde{C}_{i} & \tilde{A}_{i}^{T} \eta_{i} \tilde{B}_{i}+\tilde{C}_{i}^{T} \tilde{D}_{i} \\
* & -\left(\gamma^{2} I-\tilde{B}_{i}^{T} \eta_{i} \tilde{B}_{i}-\tilde{D}_{i}^{T} \tilde{D}\right)
\end{array}\right]<0, \forall i \in I,
$$

where $\eta_{i}:=\sum_{j=1}^{N} \lambda_{i j} P_{j}$ have a resolvable matrices $P=$ 
$\left\{P_{1}, P_{2}, \ldots P_{N}\right\}$ such that the MJLS (5) with totally known TRs is randomly stable with an $H_{\infty}$ performance index $\gamma$.

\section{Main results}

In this section, based on Lemma 1, firstly, we will introduce an $H_{\infty}$ performance analysis criterion for the error augmented system (5) and further focus on the design of the FD reduced-order filter for MJLS (1) with deficient mode information.

\section{1 $H_{\infty}$ FD filter with deficient transition information performance analysis}

The following lemma presents an $H_{\infty}$ FD filter performance analysis criterion for the underlying augmented error system in (5) with deficient TRs.

Lemma 2. Let $\gamma>0$ be a given scalar; if there are positive-definite symmetric matrices $P=\left\{P_{1}, P_{2}, \ldots P_{N}\right\}$ such that LMI (8) holds, then the error augmented system in (5) with deficient transition information is randomly stable with a guaranteed $H_{\infty}$ performance index $\gamma$ and satisfies (6).

$$
\begin{aligned}
& {\left[\begin{array}{cccc}
-\left(\eta_{j}^{(i s)}\right)^{-1} & 0 & \tilde{A}_{i} & \tilde{B}_{i} \\
* & -I & \tilde{C}_{i} & \tilde{D}_{i} \\
* & * & -P_{i} & 0 \\
* & * & * & -\gamma^{2} I
\end{array}\right]<0} \\
& \eta_{j}^{(i s)}:=\sum_{j \in S_{k}^{(i)}} \lambda_{i j} P_{j}+\sum_{j \in S_{u c}^{(i)}} \tilde{\lambda}_{i j}^{(s)} P_{j}+\sum_{j \in S_{u k}^{(i)}} \hat{\lambda}_{u k} P_{j}, \\
& \sum_{j \in S_{u k}^{(i)}} \hat{\lambda}_{u k}:=1-\sum_{j \in S_{k}^{(i)}} \lambda_{i j}-\sum_{j \in S_{u c}^{(i)}} \tilde{\lambda}_{i j}^{(s)} .
\end{aligned}
$$

Proof. By virtue of Lemma 1, it is shown that system (5) with totally known TRs is randomly stable with an $H_{\infty}$ performance $\gamma$, when matrix inequality (7) holds. Now due to $\Sigma_{j=1}^{N} \lambda_{i j}=1$ and with deficient TRs, we rewrite the term

$$
\Sigma_{j=1}^{N} \lambda_{i j} P_{j}=\sum_{j \in S_{k}^{(i)}} \lambda_{i j} P_{j}+\sum_{j \in S_{u c}^{(i)}}\left(\sum_{s=1}^{M} \alpha_{s} \tilde{\lambda}_{i j}^{(s)}\right) P_{j}+\sum_{j \in S_{u k}^{(i)}} \hat{\lambda}_{u k} P_{j} .
$$

Considering the fact that $0 \leq \alpha_{s} \leq 1, \sum_{s=1}^{M} \alpha_{s}=1$, (9) can be rewritten as

$$
\begin{aligned}
\Sigma_{j=1}^{N} \lambda_{i j} P_{j} & =\sum_{s=1}^{M} \alpha_{s} \\
& \left(\sum_{j \in S_{k}^{(i)}} \lambda_{i j} P_{j}+\sum_{j \in S_{u c}^{(i)}} \tilde{\lambda}_{i j}^{(s)} P_{j}+\sum_{j \in S_{u k}^{(i)}} \hat{\lambda}_{u k} P_{j}\right)=\eta_{j}^{(i s)} .
\end{aligned}
$$

Thus, with deficient transition information, (7) can be rewritten as

$$
\begin{aligned}
& {\left[\begin{array}{cc}
\tilde{A}_{i}^{T} \eta_{i} \tilde{A}_{i}-P_{i}+\tilde{C}_{i}^{T} \tilde{C}_{i}^{\tilde{1}} & A_{i}^{T} \eta_{i} \tilde{B}_{i}+\tilde{C}_{i}^{T} \tilde{D}_{i} \\
* & -\left(\gamma^{2} I-\tilde{B}_{i}^{T} \eta_{i} \tilde{B}_{i}-\tilde{D}_{i}^{T} \tilde{D}_{i}\right)
\end{array}\right]} \\
& =\sum_{s=1}^{M} \alpha_{s} \\
& {\left[\begin{array}{cc}
\tilde{A}_{i}^{T} \eta_{j}^{(i s)} \tilde{A}_{i}-P_{i}+\tilde{C}_{i}^{T} \tilde{C}_{i} & \tilde{A}_{i}^{T} \eta_{j}^{(i s)} \tilde{B}_{i}+\tilde{C}_{i}^{T} \tilde{D}_{i} \\
* & -\left(\gamma^{2} I-\tilde{B}_{i}^{T} \eta_{j}^{(i s)} \tilde{B}_{i}-\tilde{D}_{i}^{T} \tilde{D}_{i}\right)
\end{array}\right] .}
\end{aligned}
$$

Then, the right-hand side (RHS) of equality (10) can be further processed as

$$
\begin{aligned}
& \operatorname{RHS}(10)= \\
& {\left[\begin{array}{cc}
\tilde{A}_{i}^{T} \eta_{j}^{(i s)} \tilde{A}_{i}-P_{i}+\tilde{C}_{i}^{T} \tilde{C}_{i} & \tilde{A}_{i}^{T} \eta_{j}^{(i s)} \tilde{B}_{i}+\tilde{C}_{i}^{T} \tilde{D}_{i} \\
* & -\left(\gamma^{2} I-\tilde{B}_{i}^{T} \eta_{j}^{(i s)} \tilde{B}_{i}-\tilde{D}_{i}^{T} \tilde{D}_{i}\right)
\end{array}\right] .}
\end{aligned}
$$

The RHS of equality (11) can be decomposed into the following form:

$$
\begin{aligned}
\operatorname{RHS}(11)= & {\left[\begin{array}{ccc}
\tilde{A}_{i}^{T} \eta_{j}^{(i s)} & \tilde{A}_{i} & \tilde{A}_{i}^{T} \eta_{j}^{(i s)} \tilde{B}_{i} \\
* & \tilde{B}_{i}^{T} \eta_{j}^{(i s)} \tilde{B}_{i}
\end{array}\right] } \\
& +\left[\begin{array}{cc}
-P_{i}+\tilde{C}_{i}^{T} \tilde{C}_{i} & \tilde{C}_{i}^{T} \tilde{D}_{i} \\
* & -\gamma^{2} I+\tilde{D}_{i}^{T} \tilde{D}_{i}
\end{array}\right] \\
= & {\left[\begin{array}{cc}
\tilde{A}_{i}^{T} \eta_{j}^{(i s)} \tilde{A}_{i} & \tilde{A}_{i}^{T} \eta_{j}^{(i s)} \tilde{B}_{i} \\
* & \tilde{B}_{i}^{T} \eta_{j}^{(i s)} \tilde{B}_{i}
\end{array}\right] } \\
& +\left[\begin{array}{cc}
\tilde{C}_{i}^{T} \tilde{C}_{i} & \tilde{C}_{i}^{T} \tilde{D}_{i} \\
* & \tilde{D}_{i}^{T} \tilde{D}_{i}
\end{array}\right]+\left[\begin{array}{cc}
-P_{i} & 0 \\
* & -\gamma^{2} I
\end{array}\right]
\end{aligned}
$$

In fact, by Schur complement, RHS (12) is equivalent to

$$
\begin{aligned}
\operatorname{RHS}(12)= & {\left[\begin{array}{ccc}
-I & \tilde{C}_{i} & \tilde{D} \\
\tilde{C}_{i}^{T} & -P_{i} & 0 \\
\tilde{D}_{i}^{T} & 0 & -\gamma^{2} I
\end{array}\right]+\left[\begin{array}{ccc}
\tilde{A}_{i}^{T} \eta_{j}^{(i s)} & \tilde{A}_{i} & \tilde{A}_{i}^{T} \eta_{j}^{(i s)} \tilde{B}_{i} \\
& * & \tilde{B}_{i}^{T} \eta_{j}^{(i s)} \tilde{B}_{i}
\end{array}\right] } \\
= & {\left[\begin{array}{cccc}
-\left(\eta_{j}^{(i s)}\right)^{-1} & 0 & \tilde{A}_{i} & \tilde{B}_{i} \\
* & -I & \tilde{C}_{i} & \tilde{D}_{i} \\
* & * & -P_{i} & 0 \\
* & * & * & -\gamma^{2} I
\end{array}\right]<0 . }
\end{aligned}
$$

This completes the proof of the lemma.

Remark 2. Lemma 2 presents an $H_{\infty}$ performance analysis criterion for a family of MJLSs with deficient TRs. However, it is shown that there are coupling terms in the system matrices inequality (8), which structural constraint 
significantly augments the level of design conservatism. Thus, it incurs some difficulties for fault detection filter synthesis problem. To overcome these difficulties, the slack matrix method can be adopted here in order to obtain the following improved criterion for augmented error system (5).

\subsection{Design of $H_{\infty}$ FD reduced-order filter}

The next step is to translate the $H_{\infty}$ FD reduced-order filter design problem into a model-matching problem. In the following theorem, a sufficient condition is provided for the existence of an admissible $H_{\infty}$ FD reduced-order filter with the deficient transition probabilities (3).

Theorem 1. Consider system (1) with deficient transition information, for given $\gamma>0$, determine the matrices $A_{F i}=M_{i(2)}^{-1} \tilde{A}_{i}, \quad B_{F i}=M_{i(2)}^{-1} \tilde{B}_{i}, \quad C_{F i}=\tilde{C}_{i}, D_{F i}=\tilde{D}_{i}$, then the FD reduced-order filter (4) is found so that the augmented error system (5) is randomly stable with an $H_{\infty}$ performance index $\gamma$, if there exist positive-definite symmetric matrices $P_{i}=\left[\begin{array}{cc}P_{i(1)} & P_{i(2)} \\ * & P_{i(3)}\end{array}\right] \epsilon$ $R^{\left(n_{x}+n_{r}\right) \times\left(n_{x}+n_{r}\right)}, \quad M_{i}=\left[\begin{array}{cc}M_{i(1)} & T M_{i(2)} \\ * & M_{i(3)}\end{array}\right] \in$ $R^{\left(n_{x}+n_{r}\right) \times\left(n_{x}+n_{r}\right)}$, and $\tilde{A}_{r i}, \tilde{B}_{r i}, \tilde{C}_{r i}, \tilde{D}_{r i}, \forall i \in I$, satisfy the following LMIs:

$$
\left[\begin{array}{cccccc}
\Theta_{1} & \Theta_{2} & 0 & \Theta_{4} & T M_{i(2)} A_{F i} & \Theta_{6} \\
* & \Theta_{3} & 0 & \Theta_{5} & M_{i(2)} A_{F i} & \Theta_{7} \\
* & * & -I & D_{F i} C_{i} & C_{F i} & \Theta_{8} \\
* & * & * & -P_{i(1)} & -P_{i(2)} & 0 \\
* & * & * & * & -P_{i(3)} & 0 \\
* & * & * & * & * & -\gamma^{2} I
\end{array}\right]<0,
$$

where

$$
\begin{aligned}
\eta_{j(m)}^{(i s)} & :=\sum_{j \in S_{k}^{(i)}} \lambda_{i j} P_{j(m)}+\sum_{j \in S_{u c}^{(i)}} \tilde{\lambda}_{i j}^{(s)} P_{j(m)}+\sum_{j \in S_{u k}^{(i)}} \hat{\lambda}_{u k} P_{j(m)}, \\
m & =1,2,3, \sum_{j \in S_{u k}^{(i)}} \hat{\lambda}_{u k}:=1-\sum_{j \in S_{k}^{(i)}} \lambda_{i j}-\sum_{j \in S_{u c}^{(i)}} \tilde{\lambda}_{i j}^{(s)},
\end{aligned}
$$

$$
\begin{aligned}
& \Theta_{1}=\eta_{j(1)}^{(i s)}-M_{i(1)}-M_{i(1)}^{T}, \\
& \Theta_{2}=\eta_{j(2)}^{(i s)}-H M_{i(2)}-M_{i(3)}^{T}, \\
& \Theta_{3}=\eta_{j(3)}^{(i s)}-M_{i(2)}-M_{i(2)}^{T}, \\
& \Theta_{4}=M_{i(1)} A_{i}+T M_{i(2)} B_{F i} C, \\
& \Theta_{5}=M_{i(3)} A_{i}+M_{i(2)} B_{F i} C_{i}, \\
& \Theta_{6}=\left[\begin{array}{ll}
M_{i(1)} B_{i} M_{i(1)} E_{i}+T M_{i(2)} B_{F i} D M_{i(1)} F_{i}+T M_{i(2)} B_{F i} H_{i}
\end{array}\right], \\
& \Theta_{7}=\left[\begin{array}{ll}
M_{i(3)} B_{i} & M_{i(3)} E_{i}+M_{i(2)} B_{F i} D_{i} M_{i(3)} F_{i}+M_{i(2)} B_{F i} H_{i}
\end{array}\right], \\
& \Theta_{8}=\left[\begin{array}{lll}
0 & D_{F i} D_{i} & D_{F i} H_{i}-I
\end{array}\right] .
\end{aligned}
$$

Proof. Based on Lemma 2, accomplishing a congruence transformation with (8) using given matrix $\left\{\begin{array}{llll}M_{i} & I & I & I\end{array}\right\}$ yields the following inequality

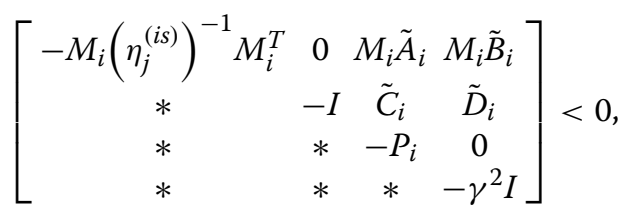

For an discretionary matrix $M_{i}, \forall i \in I$, we have the following inequality established:

$$
\left(\eta_{j}^{T}-M_{i}^{T}\right) \eta_{j}^{-1}\left(\eta_{j}-M_{i}^{T}\right) \geq 0 .
$$

We expand (17), then, get the following bounding inequality:

$$
\eta_{j}^{T}-M_{i}^{T}-M_{i}^{T} \geq-M_{i}^{T} \eta_{j}^{-1} M_{i}^{T} .
$$

It can be easily obtained the following form:

$$
\left[\begin{array}{cccc}
\eta_{j}^{(i s)}-M_{i}-M_{i}^{T} & 0 & M_{i} \tilde{A}_{i} & M_{i} \tilde{B}_{i} \\
* & -I & \tilde{C}_{i} & \tilde{D}_{i} \\
* & * & -P_{i} & 0 \\
* & * & * & -\gamma^{2} I
\end{array}\right]<0 .
$$

In (19), in order to further research, we assume that the matrix $P, M$ have the following forms:

$$
P_{i}:=\left[\begin{array}{cc}
P_{i(1)} & P_{i(2)} \\
* & P_{i(3)}
\end{array}\right], M_{i}:=\left[\begin{array}{cc}
M_{i(1)} & M_{i(2)} \\
* & M_{i(3)}
\end{array}\right], i \in I .
$$

For $H_{\infty}$ FD filter design purpose, we choose the slack matrix $M_{i}$ as:

$$
M_{i}:=\left[\begin{array}{cc}
M_{i(1)} & T M_{(2)} \\
M_{i(3)} & M_{(4)}
\end{array}\right], i \in I,
$$

where

$$
\begin{aligned}
& T:=\left[I_{n_{r}} O_{n_{r} \times\left(n_{x}-n_{r}\right)}\right]^{T}, M_{i(1)} \in R^{n_{x} \times n_{x}}, M_{i(3)} \in R^{n_{r} \times n_{x}}, \\
& M_{(2)} \in R^{n_{r} \times n_{r}}, \text { and } M_{(4)} \in R^{n_{r} \times n_{r}} .
\end{aligned}
$$

Then, according to formula (21), performing the following congruent transformation

$$
\left[M_{i}+M_{i}^{T}\right]=\left[\begin{array}{cc}
M_{i(1)}+M_{i(1)}^{T} & T M_{(2)}+M_{i(3)}^{T} \\
* & M_{(4)}+M_{(4)}^{T}
\end{array}\right],
$$

by $\left[\begin{array}{lc}I & 0 \\ * & M_{(2)} M_{(4)}^{-1}\end{array}\right]$ yields 


$$
\begin{aligned}
& {\left[\begin{array}{ll}
I & 0 \\
* & M_{(2)} M_{(4)}^{-1}
\end{array}\right]\left[\begin{array}{cc}
M_{i(1)}+M_{i(1)}^{T} & T M_{(2)}+M_{i(3)}^{T} \\
M_{i(3)} & M_{(4)}+M_{(4)}^{T}
\end{array}\right]\left[\begin{array}{ll}
I & 0 \\
* & M_{(4)}^{-T} M_{(2)}^{T}
\end{array}\right]} \\
& =\left[\begin{array}{cc}
M_{i(1)}+M_{i(1)}^{T} & T M_{(2)} M_{(4)}^{-T} M_{(2)}^{T}+M_{i(3)}^{T} M_{(4)}^{-T} M_{(2)}^{T} \\
* & M_{(2)} M_{(4)}^{-T} M_{(2)}^{T}+M_{(2)} M_{(4)}^{-1} M_{(2)}^{T}
\end{array}\right] \\
& =\left[\begin{array}{cc}
M_{i(1)}+M_{i(1)}^{T} & T M_{i(2)}+M_{i(3)} \\
* & M_{i(2)}+M_{i(2)}^{T}
\end{array}\right] .
\end{aligned}
$$

Thus, matrix $M_{i}$ in (21) can been directly specified the following general form:

$$
M_{i}:=\left[\begin{array}{cc}
M_{i(1)} & T M_{i(2)} \\
M_{i(3)} & M_{i(2)}
\end{array}\right], i \in I
$$

It is shown that in this way the matrix variable $M_{i(2)}$ can be absorbed by the filter model gain variables $A_{F i}$ and $B_{F i}$ by introducing

$$
\tilde{A}_{i}:=M_{i(2)} A_{F i}, \tilde{B}_{i}:=M_{i(2)} B_{F i} .
$$

This feature enables one to make no congruent transformation to the original matrix inequality, and all the slack variables can be set as Markov switching. Further define matrix variables

$$
\tilde{C}_{i}=C_{F i}, \tilde{D}_{i}=D_{F i}, \eta_{j}^{(i s)}=\left[\begin{array}{cc}
\eta_{1 j}^{(i s)} & \eta_{2 j}^{(i s)} \\
* & \eta_{3 j}^{(i s)}
\end{array}\right] .
$$

Then, we replace matrices $M_{i}$ given by (24) into (19); together with the admissible filter parameter, matrices are defined in (25) and (26). Finally, we can get (14) exactly. This completes the proof.

Remark 3. Up until now, it has shown that the main result presented in Theorem 1 that not only provides performance index $\gamma^{*}$ but also gives a numerically efficient and reliable approach to determine the corresponding gains of an admissible FD reduced-order filter in (4) by using Matlab software. In order to acquire a receivable $H_{\infty}$ FD reduced-order filter with $\gamma$ made as small as possible in (6), it is necessary to calculate the inequality (14) in Theorem 1 iteratively. Also, it can be derived from (14) that the design FD reduced-order filter and the corresponding error between residual and fault should be different on the basis of the different degree of deficient statistics of mode transitions. The main goal is to make the error as small as possible. To illustrate the feasibility and effectiveness of the proposed FD scheme, a numerical example will be given in the next section.

\section{Numerical example}

For simplicity, we only consider the addressed FD problem for a discrete-time MJLS with deficient transition information. Consider MJLS (1) with four operation modes, and the following matrices:

$$
\begin{aligned}
& A_{1}=\left[\begin{array}{cccc}
0.05 & -0.27 & 0.44 & 0.39 \\
0.55 & 0.33 & 0.38 & 0.55 \\
0.1 & 0.17 & 0.27 & 0.44 \\
0.05 & 0.22 & 0.16 & 0.11
\end{array}\right], \\
& A_{2}=\left[\begin{array}{cccc}
0.11 & -0.17 & 0.27 & 0.44 \\
0.55 & 0.06 & 0.22 & 0.55 \\
0.05 & 0.17 & 0.28 & 0.44 \\
0.17 & 0.05 & 0.06 & -0.11
\end{array}\right] \text {, } \\
& \begin{aligned}
A_{3} & =\left[\begin{array}{cccc}
0.16 & 0.06 & -0.02 & 0.18 \\
0.04 & -0.37 & 0.53 & -0.04 \\
-0.08 & -0.32 & -0.05 & -0.11 \\
-0.17 & 0.4 & 0.04 & 0.29
\end{array}\right], \\
A_{4} & =\left[\begin{array}{cccc}
0.23 & 0.01 & -0.55 & -0.38 \\
-0.33 & 0.36 & -0.48 & -0.1 \\
-0.20 & -0.45 & 0.1 & -0.19 \\
0.23 & 0.16 & 0.5 & -0.3
\end{array}\right],
\end{aligned}
\end{aligned}
$$

$B_{1}=[0.5 ; 0.2 ; 0.3 ; 0.1], B_{2}=[-0.8 ;-0.2 ;-0.1 ;-1]$, $B_{3}=[0.2 ;-0.2 ;-0.1 ; 0.1], B_{4}=[0.9 ;-1.1 ;-0.7 ;-1.2]$, $C_{1}=\left[\begin{array}{llll}1 & 0.1 & 0.2 & -0.3\end{array}\right], C_{2}=\left[\begin{array}{llll}0.5 & -0.8 & 0.3 & 0.5\end{array}\right]$, $C_{3}=\left[\begin{array}{llll}1.4 & 0.7 & 0.2 & -0.8\end{array}\right], C_{4}=\left[\begin{array}{lll}-0.71 .2 & 1.2 & -0.6\end{array}\right]$, $D_{1}=D_{2}=D_{3}=D_{4}=0.5$,

$E_{1}=E_{2}=E_{3}=E_{4}=[0.08 ; 0.12 ; 0.50 ; 0.29]$,

$F_{1}=F_{2}=F_{3}=F_{4}=[1 ;-1 ;-1 ; 1]$, $H_{1}=H_{2}=H_{3}=H_{4}=[-1]$.

In order to make the simulation simplification, we consider the known control input $u(k)$ is simulated by step signal with amplitude 0.2 for $k=0,1,2, \ldots, 300$. The exogenous disturbance input $\omega_{k}$ is given by white noise signal with amplitude less than 0.5 . The fault signal $f_{k}$ is:

$$
f_{k}=\left\{\begin{array}{l}
2,100 \leq k \leq 200 \\
0, \text { others }
\end{array}\right.
$$

Now, four cases for different transition rate matrix (TRM) are shown in Table 1, and the simulation result of Markov chain $r_{k}$ is given in Fig. 1 .

Table 1 Four different TRMs

\begin{tabular}{ll}
\hline Case1: completely known TRM & Case 2: polytopic uncertain TRM \\
\hline$\left[\begin{array}{llll}0.3 & 0.2 & 0.1 & 0.4 \\
0.3 & 0.2 & 0.3 & 0.2 \\
0.1 & 0.5 & 0.3 & 0.1 \\
0.2 & 0.2 & 0.1 & 0.5\end{array}\right]$ & {$\left[\begin{array}{llll}0.3 & 0.2 & \hat{\lambda}_{13} & \hat{\lambda}_{14} \\
\hat{\lambda}_{21} & \hat{\lambda}_{22} & \hat{\lambda}_{23} & \hat{\lambda}_{24} \\
\tilde{\lambda}_{31} & \hat{\lambda}_{32} & \tilde{\lambda}_{33} & \hat{\lambda}_{34} \\
\hat{\lambda}_{41} & \hat{\lambda}_{42} & 0.1 & 0.5\end{array}\right]$}
\end{tabular}

Case 3: partly known TRM

Case4: completely unknown TRM

$\left[\begin{array}{llll}0.3 & 0.2 & \hat{\lambda}_{13} & \hat{\lambda}_{14} \\ \hat{\lambda}_{21} & \hat{\lambda}_{22} & 0.3 & \hat{\lambda}_{24} \\ \hat{\lambda}_{31} & \hat{\lambda}_{32} & \hat{\lambda}_{33} & \hat{\lambda}_{34} \\ \hat{\lambda}_{41} & \hat{\lambda}_{42} & 0.1 & 0.5\end{array}\right] \quad\left[\begin{array}{llll}\hat{\lambda}_{11} & \hat{\lambda}_{12} & \hat{\lambda}_{13} & \hat{\lambda}_{14} \\ \hat{\lambda}_{21} & \hat{\lambda}_{22} & \hat{\lambda}_{23} & \hat{\lambda}_{24} \\ \hat{\lambda}_{31} & \hat{\lambda}_{32} & \hat{\lambda}_{33} & \hat{\lambda}_{34} \\ \hat{\lambda}_{41} & \hat{\lambda}_{42} & \hat{\lambda}_{43} & \hat{\lambda}_{44}\end{array}\right]$




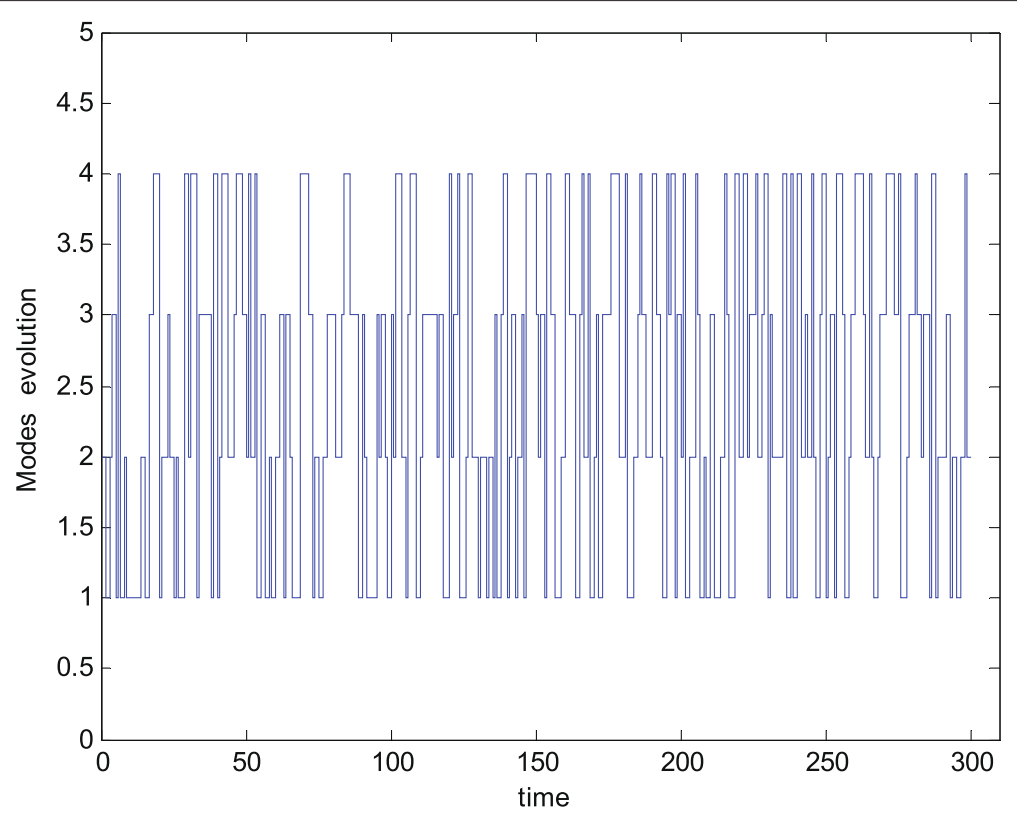

Fig. 1 Modes evolution

For case 2, the TRM includes three vertices $\Delta_{r}, r=$ $1,2,3$, and their third row $\Delta_{r}^{3}, r=1,2,3$, are given by

$$
\begin{aligned}
& \Delta_{1}^{3}=\left[\begin{array}{llll}
0.5 & \hat{\lambda}_{32} & 0.2 & \hat{\lambda}_{34}
\end{array}\right],
\end{aligned}
$$

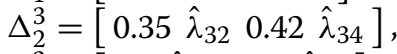

$$
\begin{aligned}
& \Delta_{3}^{3}=\left[\begin{array}{llll}
0.2 & \hat{\lambda}_{32} & 0.36 & \hat{\lambda}_{34}
\end{array}\right] \text {. }
\end{aligned}
$$
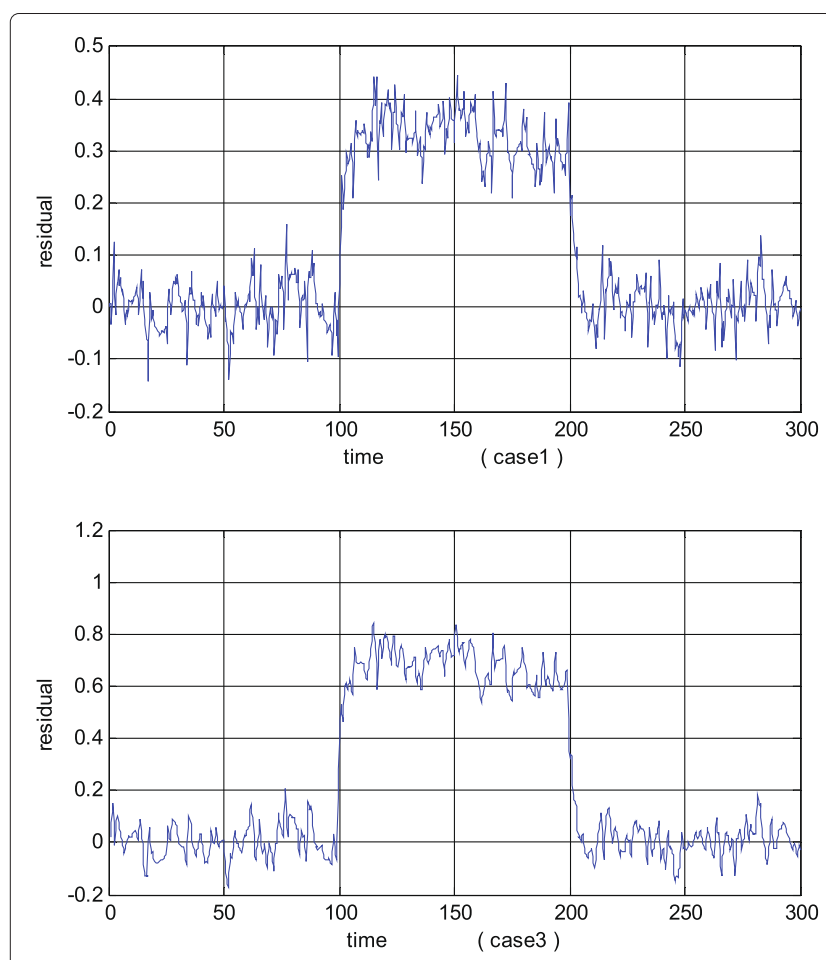

Fig. 2 Generated residual
Applying Theorem 1 through the Matlab LMI Toolbox, the gains of an admissible FD filter in the form of (4) for four different TRMs in Table 1 are acquired respectively.

Obviously, it is seen from Fig. 2, which presents the generated residual signals $\hat{r}_{k}$, that the more transition rate information we have known, the smaller the generated
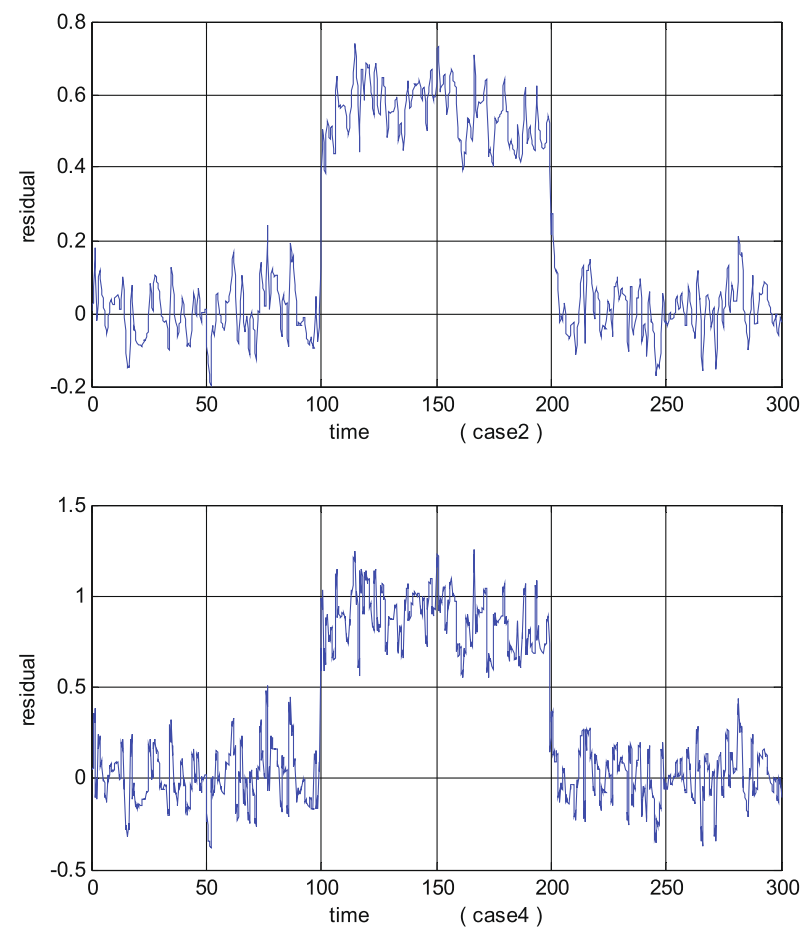

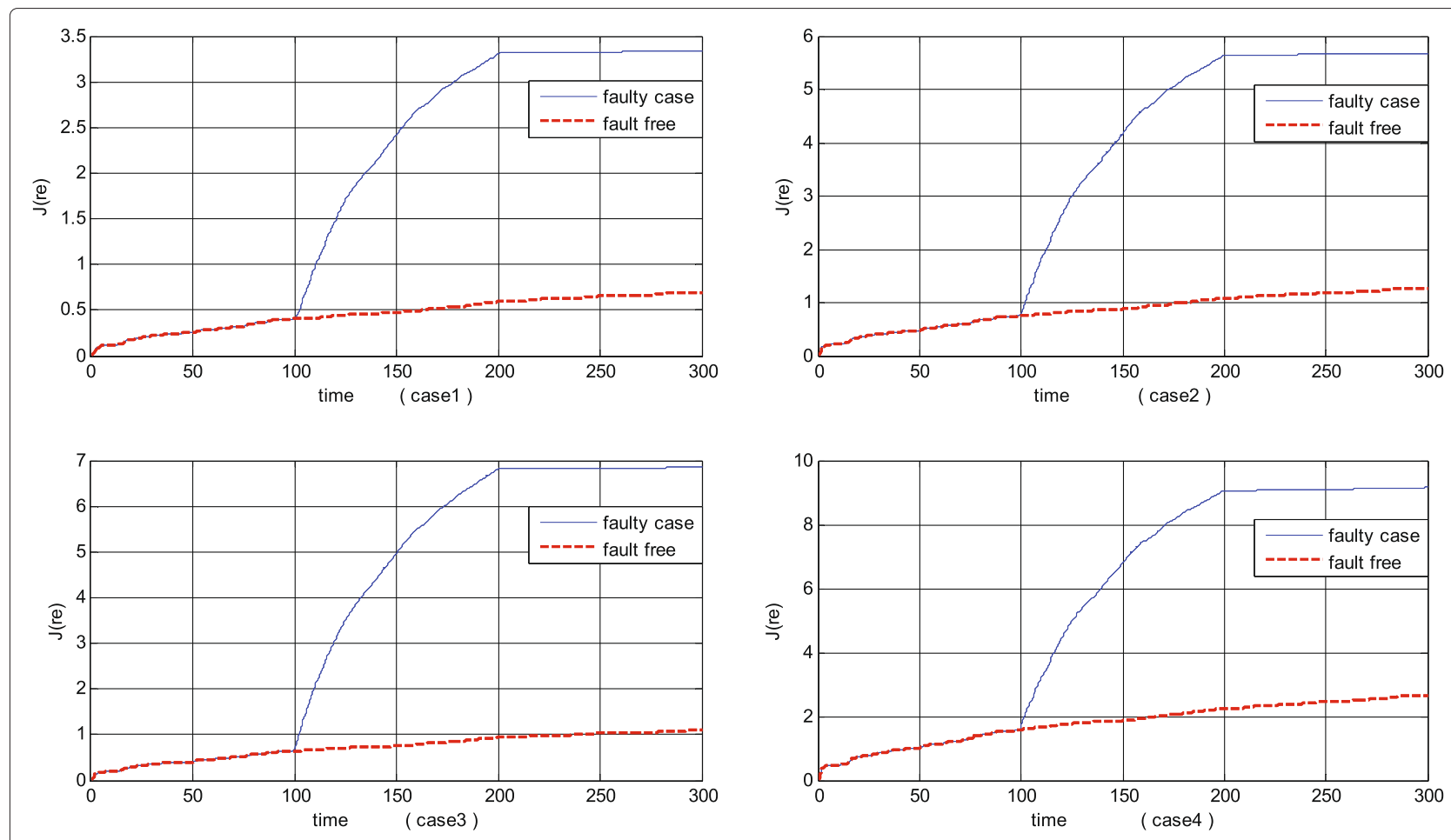

Fig. 3 Evolution of $\mathrm{J}(\mathrm{rF})$

residual $\hat{r}_{k}$ will become, e.g., the generated residual value in case 2 is the smaller than the residual value in case 3. The simulation results of polytopic uncertain effect better than partly known and completely unknown.

In the following, Fig. 3 displays the evolution of $J\left(\hat{r}_{k}\right)=$ $\sqrt{\sum_{k=k_{0}}^{k_{0}+L} \hat{r}_{k}^{T} \hat{r}_{k}}$ for both faulty case and fault-free case, respectively. It can be concluded from Fig. 3 that, when the fault occurs, the residual and the residual evaluation function have obvious change and the $H_{\infty}$ performance indices for the error augmented system (5) in case 2 are better than those in case 3 and case 4 .

According to the path in Fig. 1 and the residual threshold $J_{\text {th }}=\sup _{d \in l_{2}, f=0} E\left[\sqrt{\sum_{k=k_{0}}^{k_{0}+L} \hat{r}_{k}^{T} \hat{r}_{k}}\right]$, for the four different TRM cases, the optimal $H_{\infty}$ performance indices and the corresponding time steps for the FD are obtained

Table 2 Computation results for four different reduced-order FD filter cases

\begin{tabular}{lll}
\hline Transition rate matrix & $J_{\min }$ & Time steps \\
\hline Completely known (case 1) & 2.0057 & 102 \\
Polytopic uncertain (case 2) & 2.5169 & 103 \\
Partly known (case 3) & 2.6306 & 105 \\
Completely unknown (case 4) & 3.3299 & 107
\end{tabular}

in Tables 2 and 3. The filter gain is set to 0.1. From the computation results, it can be also shown that the FD capability in case 2 is stronger than that in case 3 and case 4. From the comparison results of the same-order FD filters, it is clear to see that the fault detection results in polytopic uncertain TRs are less conservative than those in incompletely known and completely unknown TRs. The more polytopic uncertain knowledge in the TRM, the faster sensitivity to faults will be taken and the better fault detection performance the filter can attain. The time steps to detect the fault have been shortened. Finally, comparing Tables 2 and 3, we can find that FD speed with the reduced-order filter is faster than that with full-order filter in the same case. Thus, it declares the effectiveness of the designed FD reduced-order filter for MJLSs with deficient transition information.

Table 3 Computation results for four different full-order FD filter cases

\begin{tabular}{lll}
\hline Transition rate matrix & $J_{\min }$ & Time steps \\
\hline Completely known (case 1) & 2.0032 & 104 \\
Polytopic uncertain (case 2) & 2.4527 & 106 \\
Partly known (case 3) & 2.5062 & 107 \\
Completely unknown (case 4) & 3.2015 & 109 \\
\hline
\end{tabular}




\section{Conclusion}

In this paper, a fault detection approach is proposed for discrete-time MJLSs with deficient transition information. The main contribution of our study is the introduction of Markov jump system with deficient transition information in fault detection reduced-order filter design. Special emphasis is polytopic uncertain entries have been included in deficient transition information. The underlying systems are more general, where the deficient transition descriptions are assumed to be completely known, polytopic uncertain, partly unknown, and completely unknown transition rates. Based on the linear matrix inequality approach and the linear convex optimization, a sufficient condition of FD reduced-order filter for MJLS with deficient transition information is obtained, such that the augmented error system is stochastically stable. Then, the changes of the fault signal approximately equal to the changes of the residual signal. Finally, a numerical simulation example has been given to illustrate the effectiveness of the proposed design approach. An interesting topic for future works includes fault detection and faulttolerant control methods for nonhomogeneous Markov process in the nonlinear systems.

Competing interests

The authors declare that they have no competing interests.

\section{Acknowledgements}

This work was supported by the National Natural Science Foundation of China (grant number 61503091) and the China Postdoctoral Science Foundation (2015M570282) http://www.ctan.org/tex-archive/macros/latex/contrib/ elsarticle.

\section{Author details}

${ }^{1}$ College of Automation, Harbin Engineering University, Harbin 150001, China. ${ }^{2}$ College of Information Technology, Heilongjiang Bayi Agricultural University, Daqing 163319, China.

Received: 24 February 2016 Accepted: 15 June 2016 Published online: 29 June 2016

\section{References}

1. P Shi, EK Boukas, RK Agarwal, Control of Markovian jump discrete-time systems with norm bounded uncertainty and unknown delay. IEEE Transac. Auto. Cont. 44(11), 2139-2144 (1999)

2. J Qiu, Y Wei, H Karimi, New approach to delay-dependent $H_{\infty}$ control for continuous-time Markovian jump systems with time-varying delay and deficient transition descriptions. J. Franklin Inst. 352(1), 189-215 (2015)

3. Y Wei, J Qiu, H. R Karimi, Quantized $H_{\infty}$ Filtering for Continuous-Time Markovian Jump Systems with Deficient Mode Information. Asian Journal of Control. 17, 1914-1923 (2015)

4. J Qiu, H Tian, Q Lu, H Gao, Non-synchronized robust filtering design for continuous-time T-S fuzzy affine dynamic systems based on piecewise Lyapunov functions. IEEE Trans. on Cybernetics. 43(6), 1755-1766 (2013)

5. Y Wei, J Qiu, HR Karimi, M Wang, A new design of $H_{\infty}$ filtering for continuous-time Markovian jump systems with time-varying delay and partially accessible mode information. Signal Process. 93(9), 2392-2407 (2013)

6. M Zhong, $\mathrm{H}$ Ye, P Shi, Fault detection for Markovian jump systems. IEE Proc Control Theory Appl. 152(4), 397-402 (2005)

7. J Qiu, G Feng, H Gao, Observer-based piecewise affine output feedback controller synthesis of continuous-time T-S fuzzy affine dynamic systems using quantized measurements. IEEE Transac. Fuzzy Syst. 20(6), 1046-1062 (2012)
8. Y Wei, J Qiu, S Fu, Mode-dependent nonrational output feedback control for continuous-time semi-Markov jump systems with time-varying delay. Nonlinear Analysis-Hybrid Syst. 16, 52-71 (2015)

9. J Qiu, SX Ding, H Gao, S Yin, Fuzzy-model-based reliable static output feedback H-infinity control of nonlinear hyperbolic PDE systems. IEEE Transac Fuzzy Syst. 24(2), 388-400 (2016)

10. Y Wei, J Qiu, HR Karimi, New results on $H_{\infty}$ dynamic output feedback control for Markovian jump systems with time-varying delay and deficient mode information. Optimal Control Appl. Methods. 35(6), 656-675 (2014)

11. M Wang, J Qiu, M Chadli, M Wang, A switched system approach to exponential stabilization of sampled-data T-S fuzzy systems with packet dropouts. IEEE Trans. Cybernetics (2015). doi: 10.1109/TCYB.2015.2498522

12. W Cui, J Fang, Y Shen, W Zhang, Disipativity analysis of singular systems with Markovian jump parameters and mode-dependent mixed time-delays. Neurocomputing. 110, 121-127 (2013)

13. J Qiu, G Feng, J Yang, A new design of delay-dependent robust $H_{\infty}$ filtering for continuous-time polytopic systems with time-varying delay. Int. J. Robust Nonlinear Control. 20(3), 346-365 (2010)

14. OLV Costa, RAM GBenites, Linear minimum mean square filter for discrete-time linear systems with Markov jumps and multiplicative noises. Automatica. 47, 466-476 (2011)

15. P Bolzerna, P Colaneria, GD Nicolao, Markov Jump Linear Systems with switching transition rates: Mean square stability with dwell-time. Automatica. 46, 1081-1088 (2010)

16. Y Wei, M Wang, J Qiu, New approach to delay dependent $H_{\infty}$ filtering for discrete-time Markovian jump systems with time-varying delay and incomplete transition descriptions. IET Control Theory Appl. 7(5), 684-696 (2013)

17. J Qiu, G Feng, J Yang, Robust $H_{\infty}$ static output feedback control of discrete-time switched polytopic linear systems with average dwell-time. Sci. China Ser. F Inf. Sci. 52(11), 2019-2031 (2009)

18. Y Wei, X Peng, J Qiu, $H_{\infty}$ filtering for two-dimensional continuous-time Markovian jump systems with deficient transition descriptions. Neurocomputing. 167, 406-417 (2015)

19. I Hwang, S Kim, Y Kim, A Survey of Fault Detection, Isolation, and Reconfiguration Methods. IEEE Transac. Control Syst. Tech. 18(3), 636-653 (2010)

20. J Qiu, H Gao, SX Ding, Recent advances on fuzzy-model-based nonlinear networked control systems: A survey. IEEE Transac. Ind. Electron. 63(2), 1207-1217 (2016)

21. I Jaimoukha, Z Li, $\vee$ Papakos, A matrix factorization solution to the $H_{\infty}$ fault detection problem. Automatica. 42(11), 1907-1912 (2006)

22. L Li, SX Ding, J Qiu, Y Yang, Real-time fault detection approach for nonlinear systems and its asynchronous T-S fuzzy observer-based implementation. IEEE Transac. Cybernetics (2016). doi: 10.1109/TCYB.2015.2513438.

23. MT Raza, AQ Khan, G Mustafa, Design of Fault Detection and Isolation Filter for Switched Control Systems Under Asynchronous Switching. 24(1), 13-23 (2016)

24. Y Wei, J Qiu, HK Lam, L Wu, Approaches to T-S fuzzy-affine-model-based reliable output feedback control for nonlinear Itô stochastic systems. IEEE Transac Fuzzy Syst (2016). doi: 10.1109/TFUZZ.2016.2566810

25. G Leger, A Rueda, Low-cost digital detection of parametric faults cascaded sigma delta modulators. IEEE Transac Circuits Syst I: Reg. 56(7), 1326-1338 (2009)

26. Y Wang, SX Ding, $\mathrm{H}$ Ye, G Wang, A new fault detection scheme for networked control systems subject to uncertain time-varying delay. IEEE Transac Signal Process. 56(10), 2558-2568 (2008)

27. Z Hameeda, YS Honga, YM Choa, Condition monitoring and fault detection of wind turbines and related algorithms: A review. Renewable Sustainable Energy Rev. 13, 1-39 (2009)

28. J Qiu, G Feng, J Yang, Delay-dependent non-synchronized robust $H_{\infty}$ state estimation for discrete-time piecewise linear delay systems. Int. J. Adapt. Control Signal Process. 23(2), 1082-1096 (2009)

29. A Casavola, D Famularo, G Franze, Robust fault detection of uncertain linear sytems via quasi-LMIs. Automatica. 44(1), 289-295 (2008)

30. F Erik, N Lars1, Robust residual generation for diagnosis including a reference model for residual behavior. Automatica. 42(3), 437-446 (2006)

31. AQ Khan, SX Ding, Threshold computation for fault detection a family of discrete-time nonlinear systems. Int J Adaptive Control Signal Process. 25(5), 407-429 (2011) 
32. J Keller, S Nowakowski, M Darouach, State estimation and failure detection in singular systems. Control Theory Adv Tech. 8(4), 755-762 (1992)

33. M Zhong, SX Ding, EL Ding, Optimal fault detection for linear discrete time-varying systems. Automatica. 46(8), 1395-1400 (2010)

34. J Gertler, Fault detection and isolation using parity relations. Control Eng. Pract. 5(5), 653-661 (1997)

35. J Chen, HY Zhang, Robust detection of faulty actuators via unknown input observers. Int J Syst Sci. 22(10), 1829-1839 (1991)

36. SK Nguang, P Shi, S Ding, Fault Detection for Uncertain Fuzzy Systems: An LMI Approach. IEEE Transac Fuzzy Syst. 15(6), 1251-1262 (2007)

37. TWang, H Gao, J Qiu, A combined adaptive neural network and nonlinear model predictive control for multirate networked industrial process control. IEEE Transac Neural Netw Learn Syst. 27(2), 416-425 (2016)

38. R Iserman, Supervision, Fault - Detection and Fault - Diagnosis Methods An Introduction. Control Eng. Pract. 5(5), 639-652 (1997)

39. C Keliris, M Polycarpou, T Parisini, A Distributed Fault Detection Filtering Approach for a Class of Interconnected Continuous-Time Nonlinear Systems. IEEE Transac. Auto Control. 58(8), 2032-2047 (2013)

40. L Guo, H Wang, Fault detection and diagnosis for general stochastic systems using B-spline expansions and nonlinear filters. IEEE Transac Circuits Syst Part I : Regular Papers. 52(8), 1644-1652 (2005)

41. L Li, SX Ding, J Qiu, Y Yang, Y Zhang, Weighted fuzzy observer-based fault detection approach for discrete-time nonlinear systems via piecewise-fuzzy Lyapunov functions. IEEE Transac Fuzzy Syst (2016). doi: 10.1109/TFUZZ.2016.2514371

42. PM Frank, Fault diagnosis in dynamic systems using an alytical and knowledge-based redundancy-a survey and some new results. Automatica. 26(3), 459-474 (1990)

43. M Chadlia, A Abdob, SX Ding, $H_{-} / H_{\infty}$ fault detection filter design for discrete-time Takagi-Sugeno fuzzy system. Automatica. 49, 1996-2005 (2013)

44. F Hamelin, D Sauter, Robust fault detection in uncertain dynamic systems Automatica. 36(11), 1747-1754 (2000)

45. JH Kim, Delay-dependent robust $H_{\infty}$ filtering for uncertain discrete-time singular systems with interval time-varying delay. Automatica. 46, 591-597 (2010)

46. I Shames, AMH Teixeira, H Sandberg, Distributed fault detection for interconnected second-order systems. Automatica. 47, 2757-2764 (2011)

\section{Submit your manuscript to a SpringerOpen ${ }^{\circ}$ journal and benefit from:}

- Convenient online submission

- Rigorous peer review

- Immediate publication on acceptance

- Open access: articles freely available online

- High visibility within the field

- Retaining the copyright to your article

Submit your next manuscript at $\gg$ springeropen.com 